BULL. AUSTRAL. MATH. SOC.

$16 A 70$

VOL. 8 (1973), 181-185.

\title{
A generalization of a theorem of Wedderburn
}

\section{Steve Llgh}

Outcalt and Yaqub have extended the Wedderburn Theorem which states that a finite division ring is a field to the case where $R$ is a ring with identity in which every element is either nilpotent, or a unit. In this paper we generalize their result to the case where $R$ has a left identity and the set of nilpotent elements is an ideal. We also construct a class of non-commutative rings showing that our generalization of Outcalt and Yaqub's result is real.

\section{Introduction}

Wedderburn's Theorem, asserting that a finite division ring is necessarily commutative, has been generalized in several directions $[1,6$, $7,8]$. A survey on a few papers concerning commutativity theorems for rings revealed that the two non-commutative rings defined on the Klein group $(G,+)$ have been cited quite often as counterexamples to show that certain hypotheses cannot be deleted. In particular, one of them has the following multiplication: if $x \neq 0$ is in $G$, then $x g=0$ or $x g=g$ for all $g$ in $G$. One of the purposes of this note is to characterize the class of abelian groups which admit such multiplications. Then this class of rings is used to obtain a generalization of a theorem of Outcalt and Yaqub [8].

\section{A class of non-commutative rings}

DEFINITION. Let $(G,+)$ be an abelian group and $H$ a proper subset 
of $G$ such that $0 \neq H$. Define a multiplication * on $G$ as follows: $h * g=g$ and $x * g=0$ for each $h \in H, x \in G-H$ and $g \in G$. The multiplication * is called "trivial" if and only if $(G,+, *)$ is a ring.

We now give a complete characterization of the class of abelian groups which admit a trivial multiplication.

THEOREM 1. An abelian group $(G,+)$ admits a trivial multiplication if and only if each element of $G$ is of order two.

Proof. Suppose $(G,+, *)$ is a ring and * is trivial. Then there is $h \neq 0$ in $G$ such that $h g=g$ for each $g$ in $G$. Let $y \neq 0$ be an element of $G$. Since $(h+h) y=0$ or $y$, it follows that $(h+h) y=0$. Hence $0=(h+h) y=y+y$. Thus each element of $(G,+)$ is of order two.

Conversely, let $\left(G,,^{+}\right)$be an abelian group of which each element is of order two. Thus $G$ can be considered as a vector space over $Z_{2}$. Hence $G$ has a basis $B$. For each $x \neq 0$ in $G$, there is a positive integer $n$ such that $x=b_{1}+b_{2}+\ldots+b_{n}, b_{i} \in B$. Let $H=\{x \in G: n$ is odd $\}$. Define a multiplication $*$ on $G$ as follows: if $h \in H, h * g=g$ for each $g \in G$. For $x \in G-H, x * g=0$ for each $g \in G$. It follows from [5] or can be verified easily that $(G,+, *)$ satisfies all the axioms of a ring except perhaps the right distributive law. That the right distributive law also holds can be checked easily. Hence $(G,+, *)$ is a ring and the multiplication defined is trivial. This completes the proof of the theorem.

Observe that the class of rings just constructed has the following properties: the set of nilpotent elements is an ideal, left identities exist, $(x y)^{n}=x^{n} y^{n}$ for each element $x$ and $y$. This class of noncommutative rings serves as counterexamples to show that certain hypotheses of various commutativity theorems cannot be omitted. For example, see [3, $4,6,7,8]$.

\section{A commutativity theorem}

Many generalizations of the famous Wedderburn's Theorem have appeared 
recently. In [8] Outcalt and Yaqub provided the following:

THEOREM (Outcalt and Yaqub). Let $R$ be a ring with identity in which each element is either nilpotent or a unit in $R$. Then

(a) the set $N$ of nilpotent elements in $R$ is an ideal;

(b) if (i) $R / N$ is finite and (ii) $x \equiv y(\bmod N)$ implies that $x^{2}=y^{2}$ or both $x$ and $y$ commute with all elements of $N$,

then $R$ is commutative.

We now extend the above result to a much larger class of rings. We simply assume the ring has a left identity and the set of nilpotent elements is an ideal. But first we state another generalization of Wedderburn's Theorem given by Herste in [1].

THEOREM (Herstein). Let $R$. be a ring such that for every element $x$ in $R$ there exists an integer $n=n(x)$, and a polynomial $P(t)=P_{x}(t)$ with integer coefficients, such that $x^{n+1} P(x)=x^{n}$. If all the nilpotent elements of $R$ are in the center of $R$, then $R$ is commutative.

THEOREM 2. Let $R$ be a ring with a left identity $e$ and let the set of nilpotent elements be an ideal. If

(i) $R / N$ is finite and

(ii) $x \equiv y(\bmod N)$ implies that $x^{2}=y^{2}$ or both $x$ and $y$ commute with all elements of $N$,

then $R$ is commutative.

Proof. First we show that $e$ is also a right identity by demonstrating that $e$ is unique. Suppose there exists $w$ in $R$ such that $w r=r$ for each $r$ in $R$. Since $R / N$ is commutative, we see that $e w-w e=w-e$ is an element of $N$. Thus $w \equiv e(\bmod N)$ implies that $w=w^{2}=e^{2}=e$ or both $w$ and $e$ commute with all elements of $N$. In the latter case, we see that $e(w-e)=(w-e) e$ implies that $e=w$. Since $e$ is unique it follows [2, p. 55] that $e$ is the identity of $R$.

Next we wish to show that each element of $N$ is in the center of $R$. Since $R / N$ is finite, it is a direct sum of fields: 


$$
R / N=R_{1} / N \oplus R_{2} / N \oplus \ldots \oplus R_{j} / N
$$

Using Lemmas 1 and 2 in [8], we see that if $b_{i}+N$ is in $R_{i} / N$, then $a b_{i}=b_{i} a$ for each $a$ in $N$. Now let $a \in N$ and $b \in R$. Then $b=b_{1}+b_{2}+\ldots+b_{j}+n, n \in N$. Thus $a b=b a$, since by Lemme 1 in [8] $N$ is a commutative subring of $R$ and $a b_{i}=b_{i} a$ for $i=1, \ldots, j$. This shows that $N$ is a subset of the center of $R$.

Since $R / N$ is finite and has no nonzero nilpotent elements, it follows that for each $x$ in $R$ there is an integer $n=n(x)$ such that $x^{n}-x$ is in $N$. Hence there is an integer $k$ such that $x^{k}=x^{k+l} P(x)$. Now by Herstein's Theorem $R$ is commutative.

Recall that the class of non-commutative rings constructed in Section 2 satisfies the hypotheses of Theorem 2 except ( $i i)$. Hence we see that Outcalt and Yaqub's result is a corollary of our theorem.

Finally we remark that in Theorem 2 one can assume a right identity instead of a left identity. However, it is not known whether or not one can drop that assumption.

\section{References}

[1] I.N. Herstein, "A note on rings with central nilpotent elements", Proc. Amer. Math. Soc. 5 (1954), 620.

[2] Nathan Jacobson, Lectures in abstract algebra, Volzone I (Van Nostrand, New York, Toronto, London, 1951).

[3] E.C. Johnsen, D.L. Outcalt and Adil Yaqub, "An elementary comutativity theorem for rings", Amer. Math. Monthly 75 (1968), 288-289.

[4] J. Luh, "A commutativity theorem for primary rings", Acta Math. Acad. Sci. Hungar. 22 (1971), 211-213.

[5] J.J. Malone, Jr, "Near-rings with trivial multiplications", Amer. Math. Monthly 74 (1967), 1111-1112. 
[6] H.G. Moore, "On commutativity in certain rings", Bull. Austral. Math. Soc. 2 (1970), 107-115.

[7] D.L. Outcalt and Adil Yaqub, "A generalization of Wedderburn's Theorem", Proc. Amer. Math. Soc. 18 (1967), 175-177.

[8] D.L. Outcalt and Adil Yaqub, "A commutativity theorem for rings", Bulz. Austral. Math. Soc. 2 (1970), 95-99.

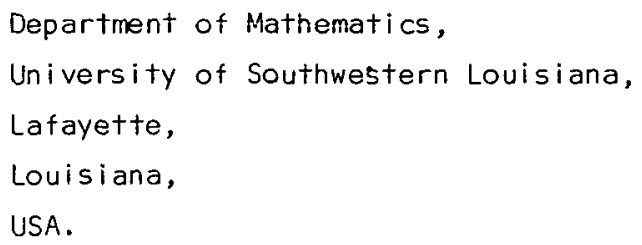

USA. 